\title{
Perfect imaging of three object points with only two analytic lens surfaces in two dimensions
}

\author{
Fabian Duerr, ${ }^{*}, a$ Pablo Benítez, ${ }^{b, c}$ Juan Carlos Miñano, ${ }^{b, c}$ Youri Meuret ${ }^{a}$ and Hugo \\ Thienpont ${ }^{a}$ \\ ${ }^{a}$ Vrije Universiteit Brussel, Faculty of Engineering Sciences, Brussels Photonics Team \\ B-PHOT, TONA-FirW, Pleinlaan 2, 1050 Brussels, Belgium \\ ${ }^{b}$ CeDInt, Universidad Politécnica de Madrid (UPM), Campus de Montegancedo, 28223 \\ Pozuelo de Alarcón, Spain \\ ${ }^{c}$ Light Prescriptions Innovators, 16662 Hale Ave., Irvine, CA USA 92606
}

\begin{abstract}
In this work, a new two-dimensional analytic optics design method is presented that enables the coupling of three ray sets with two lens profiles. This method is particularly promising for optical systems designed for wide field of view and with clearly separated optical surfaces. However, this coupling can only be achieved if different ray sets will use different portions of the second lens profile.

Based on a very basic example of a single thick lens, the Simultaneous Multiple Surfaces design method in two dimensions (SMS2D) will help to provide a better understanding of the practical implications on the design process by an increased lens thickness and a wider field of view. Fermat's principle is used to deduce a set of functional differential equations fully describing the entire optical system. The transformation of these functional differential equations into an algebraic linear system of equations allows the successive calculation of the Taylor series coefficients up to an arbitrary order. The evaluation of the solution space reveals the wide range of possible lens configurations covered by this analytic design method. Ray tracing analysis for calculated $20^{\text {th }}$ order Taylor polynomials demonstrate excellent performance and the versatility of this new analytical optics design concept.
\end{abstract}

Keywords: Mathematical methods in physics, geometric optical design and lens design, free-form optics

\section{INTRODUCTION}

In geometrical optics, the modern formulation of Fermat's principle states that rays of light between two fixed points $A$ and $B$ traverse the path of stationary optical length

$$
\delta S=\delta \int_{A}^{B} n d s=0
$$

through media with refractive index distribution $n$. As done in most textbooks on optics, this fundamental principle can be used to describe the properties of light rays refracted through different media, reflected off mirrors or undergoing total internal reflection. Indeed, all known laws of geometrical optics, lens design and aberrations will be consequences of the analytic properties of solutions to Fermat's principle. ${ }^{1}$

However, its mathematical complexity could give rise to the impression that it is impractical to be used for optics design. In fact, conventional optics design is based on minimizing a chosen merit function which quantifies the system's performance for defined sets of rays. In case of imaging applications, this can be merit functions such as the sum of the squares of certain aberrations or the RMS blur spot at the image plane. ${ }^{2}$ In case of nonimaging optics, different merit functions such as the contrast ratio at a receiver plane or the optical collection efficiency could be chosen.

For most optical design problems, perfect solutions normally do not exist. Therefore, the design strategy begins with a parameterized description of the (refractive or reflective) optical surfaces. By using multi-parametric

\footnotetext{
*E-mail correspondence: fduerr@b-phot.org; Telephone: +32 (0)2 6293570
} 
optimization (a common tool of any optical design software), these optimization algorithms normally start from an initial set of parameters and end with a final optimized set of parameters that minimizes a defined merit function. Since this is often a non-convex optimization problem, it cannot be guaranteed that these algorithms find the global minimum.

In contrast, direct optics design methods do not necessarily follow this design strategy. Usually, a key objective is to find a set of unrestricted free-form surfaces, designed to have specific predefined characteristics. Here, unrestricted means that the optical surfaces can have any shape to fulfill all imposed requirements. Such an approach can help to reduce the needed number of optical elements to a minimum or offer a way to design very compact optical systems. A basic example of such a design task is to focus light coming from a point in object space onto a point in the image space which can be achieved by using a Cartesian oval. ${ }^{3}$ In order to focus light coming from an additional object point, one surface is no longer sufficient, two surfaces are needed. In general, an optical system consisting of $N$ optical surfaces can couple $N$ sets of rays for which specific conditions are imposed. In case of optical systems designed for wide field of view and with at least one surface far from the aperture stop, it has been shown recently that it is possible to couple more than two ray sets with only two lens profiles. ${ }^{4}$ Optical systems, where different incident directions use different portions of lens' surfaces, are widely known. Field-flattener lenses are used in binocular designs and in astronomic telescopes to improve edge sharpness and lower distortion. Aperture stops in imaging systems often target the same objective.

Based on a very basic example of a single thick lens, the Simultaneous Multiple Surfaces (SMS) design method will serve as a starting point in Sec. 2. It will help to provide a better understanding of the practical implications on the design process of an increasing lens thickness and a wider field of view. The initial degrees of freedom of the SMS2D design method will lead to an irreducible design representation, based on the local coupling of two ray sets with two lens profiles parts to achieve global coupling of three ray sets with two smooth lens profiles. The concept of convergence points, which was first introduced to design free-form V-groove reflectors, ${ }^{5,6}$ will serve as a starting point for the analytic solution derived in Sec. 3. By applying Fermat's principle, it is possible to deduce a system of functional differential equations. The transformation of these functional differential equations into an algebraic linear system of equations allows the successive calculation of the Taylor series coefficients up to an arbitrary order. The obtained solution space is evaluated in Sec. 4, revealing the wide range of possible lens configurations covered by this analytic design method. Finally, ray tracing analysis is used in Sec. 5 to provide a comprehensive quantitative analysis of the convergence of the analytic design method and the direct link with the SMS2D design method. It demonstrates the versatility of this analytic design method by explicitly evaluating Taylor polynomials up to $20^{\text {th }}$ order.

\section{THE SMS2D DESIGN METHOD AND ITS INITIAL DEGREES OF FREEDOM}

The Simultaneous Multiple Surfaces (SMS) design method has proven to be very versatile in various applications. SMS surfaces are piecewise curves made of several portions of generalized Cartesian ovals that map initial ray sets to final ray sets. It involves the simultaneous calculation of $N$ optical surfaces using $N$ one-parameter ray sets for which specific conditions connect the initial with the final ray sets. ${ }^{3}$ A particular formulation of the SMS2D design method for imaging systems comprises perfect imaging of $N$ ray sets at the correspondent $N$ image points. ${ }^{7}$ The SMS2D design method for imaging systems offers the flexibility to choose the ray sets and their associated image points. ${ }^{8,9}$

SMS optics are calculated by applying a constant optical path length for each coupled ray set. In case of two lens profiles and for two design angles of opposite sign, the overall symmetry implies an identical optical path length for both ray sets. The optical path length can be determined by choosing one initial point on each lens profile. Fig. 1(a)-(c) show different SMS2D designs for $\theta= \pm 5^{\circ}$ design angles and increasing lens thicknesses from left to right. Similarly, Fig. 1(d)-(f) show SMS2D designs with increasing lens thicknesses for $\theta= \pm 10^{\circ}$ design angles. The bold light rays highlight the initial points used to determine the optical path length. Additional rays alternately construct the final lens profiles by applying the constant optical path length condition. This design process only provides a set of discrete points and correspondent normal vectors. An SMS skinning process can finally be used to fill the gaps between the separated points, fully defining both optical surfaces. Therefore, a segment can be interpolated between the two symmetric adjacent points of the bottom surface, as indicated as solid lens profile in Fig. 1. As a boundary condition, the segment has to match point coordinates and normal vectors at the initial points, which requires at least a $2^{\text {nd }}$ order polynomial function. However, in principle, any 


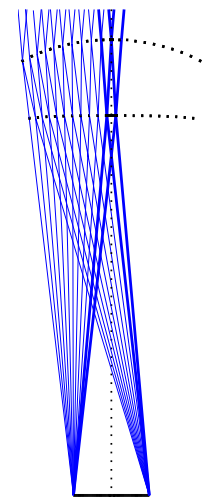

(a)

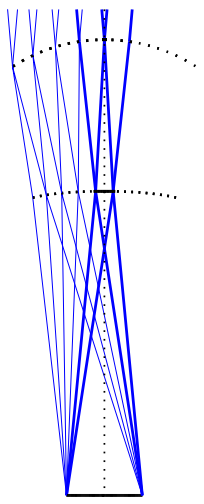

(b)

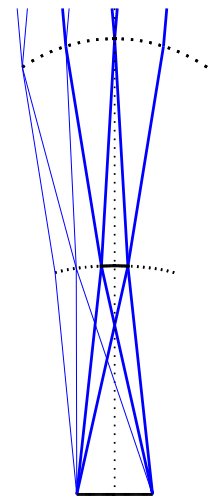

(c)

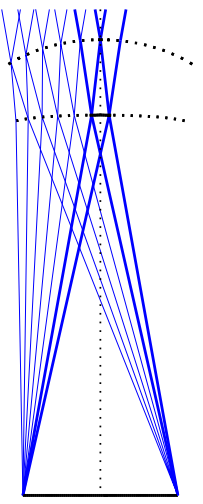

(d)

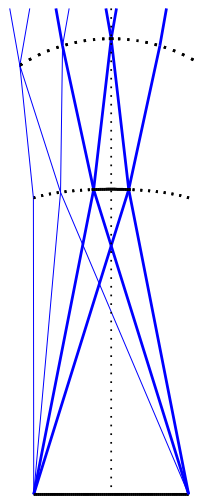

(e)

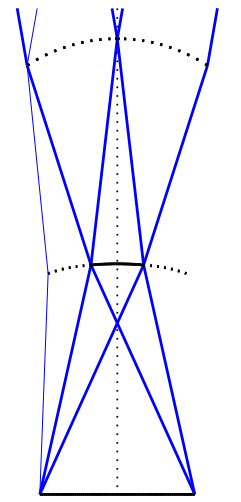

(f)

Figure 1. Impact of increasing design angles and lens thicknesses on the relative importance of the initial segment

analytical function satisfying these boundary conditions can be applied - which correspondents to an infinite parameter space for the initial segment problem. For small design angles and moderate lens thicknesses, the initial segment represents only a small fraction of the entire lens profiles. However, with increasing design angles and/or distances between the optical surfaces the relative importance of the initial segment increases as well. In extreme cases, the initial segment can completely define the full lens profiles as shown in Fig. 1(f). A selected $2^{\text {nd }}$ order polynomial segment may guarantee coupling of two ray sets with chosen design angles of opposite sign, but it does not make any further use of the full potential offered by an unrestricted initial segment satisfying additional imposed conditions. The main objective of this work is to construct initial segments which ensure maximum benefit from these infinite initial degrees of freedom.

Maximum benefit means here, that the degrees of freedom of the initial segment are used to couple an additional on-axis ray set. Consider the curve segments $\mathrm{S}_{1}$ and $\hat{\mathrm{S}}_{2}$ of the two lens profiles shown in Fig. 2(a). They are designed to couple a tilted parallel ray set onto a point. Because of the symmetry applied with respect to the optical axis of the lens, the lens segments $\hat{\mathrm{S}}_{1}$ and $\mathrm{S}_{2}$ will couple the parallel ray set with opposite sign. That means that only one ray set is actually coupled using these two surface segments. This offers the opportunity to couple an additional ray set. One of these two identical off-axis ray sets is now replaced in Fig. 2(b) by an on-axis ray set. The constant optical path length condition can then be used to calculate the final lens portion on the second lens profile by tracing off-axis rays through $\hat{\mathrm{S}}_{1}$, which is illustrated in Fig. 2(c). Finally, the full set of rays and the completed lens is shown in Fig. 2(d) - coupling three ray sets with two lens profiles.

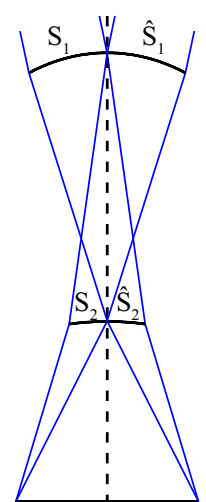

(a)

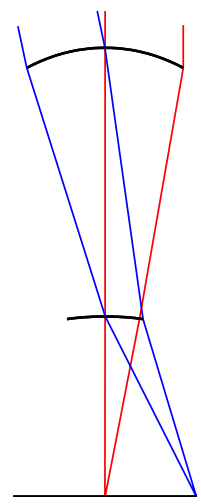

(b)

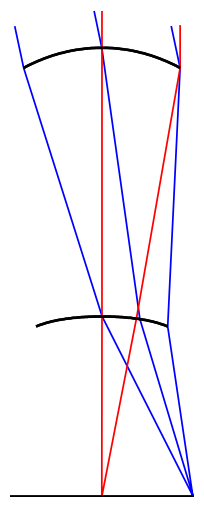

(c)

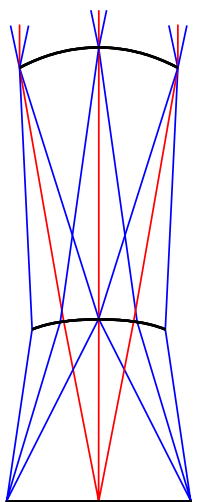

(d)

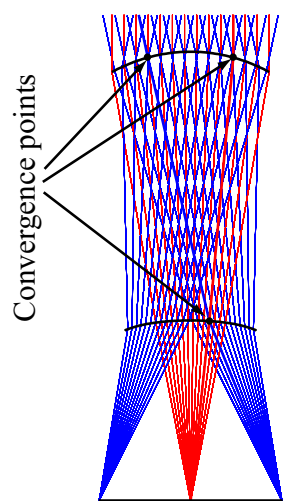

(e)

Figure 2. Illustrations (a)-(d) show how to make use of the initial segment to couple an additional on-axis ray set; illustration (e) shows the final ray paths and the highlighted convergence points 
The final ray paths are shown in Fig. 2(e). The highlighted convergence points are characterized by on- and off-axis rays sharing identical points and normal vectors on each lens profile. These convergence points will serve as a starting point to derive the analytic solution to the initial segment problem in the following section. An in detail discussion on their existence can be found in. ${ }^{4}$

\section{ANALYTIC SOLUTION TO THE STATED INITIAL SEGMENT PROBLEM}

The starting point for all further considerations is as follows: determine the convergence points first and derive the full lens profiles from these initial convergence points for one on-axis and one off-axis ray set. All necessary initial values are defined as shown in Fig. 3(a).

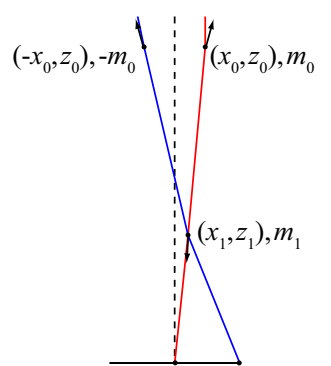

(a) $(0, d)(r, d)$

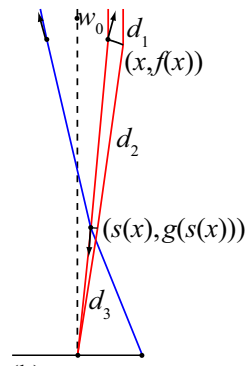

(b)

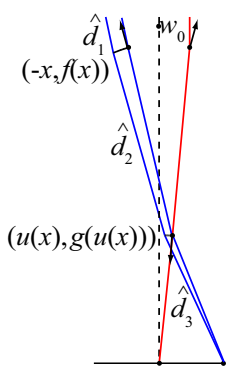

(c)

Figure 3. Shown are all necessary initial values and functions to derive the conditional equations from Fermat's principle

The point coordinates $\left(x_{0}, z_{0}\right)$ of the convergence point on the first lens profile can be freely chosen without loss of generality. The slope $m_{0}$ at the convergence point represents a first variable. The intersection of the refracted on-axis ray through $\left(x_{0}, z_{0}\right)$ and the refracted off-axis ray through the mirrored convergence point $\left(-x_{0}, z_{0}\right)$ determines the coordinates $\left(x_{1}, z_{1}\right)$ of the convergence point on the $2^{\text {nd }}$ lens profile. The slope $m_{1}$ at this second convergence point represents a second variable. The intersection of the refracted on-axis ray through $\left(x_{1}, z_{1}\right)$ and the optical axis determines the first focus position $d$. Finally, the refracted off-axis ray and the known lateral detector position determine the x-coordinate $r$ of the second focus position. This formulation provides a compact representation of all initial values depending upon the two variables $m_{0}$ and $m_{1}$ only. In addition, it provides the permissible range of values for these two variables for which the convergence point construction is possible. For example, a given value $m_{0}$ limits the possible range of values of $m_{1}$ for which an on-axis ray can be refracted towards the optical axis.

Next, two analytic functions $f(x)$ and $g(x)$ are introduced to describe the two lens profiles. To analytically describe the optical paths of rays passing through the lens it is necessary to introduce two additional mapping functions $s(x)$ and $u(x)$. Fig. 3(b) shows an on-axis ray passing through an arbitrary point $\vec{p}=(x, f(x))$ on the first lens profile which is then refracted in $(s(x), g(s(x)))$ towards the focal point $(0, d)$. The auxiliary function $s(x)$ thus defines the mapping in x-coordinate. Similarly, function $u(x)$ defines the mapping in $\mathrm{x}$-coordinate for off-axis rays through an arbitrary point $\hat{\vec{p}}=(-x, f(x))$ on the first, and through $(u(x), g(u(x)))$ on the second lens profile, as shown in Fig. 3(c). All optical path lengths can then be expressed in sections using vector geometry as

$$
\begin{aligned}
d_{1} & =\vec{v}_{0} \cdot\left(\vec{p}-\vec{w}_{0}\right) \\
d_{2} & =n_{2} \sqrt{(x-s(x))^{2}+(f(x)-g(s(x)))^{2}} \\
d_{3} & =\sqrt{s(x)^{2}+(d-g(s(x)))^{2}}
\end{aligned}
$$

for on-axis rays, and as

$$
\begin{aligned}
& \hat{d}_{1}=\vec{v}_{1} \cdot\left(\hat{\vec{p}}-\vec{w}_{0}\right) \\
& \hat{d}_{2}=n_{2} \sqrt{(-x-u(x))^{2}+(f(x)-g(u(x)))^{2}} \\
& \hat{d}_{3}=\sqrt{(r-u(x))^{2}+(d-g(u(x)))^{2}}
\end{aligned}
$$


for off-axis rays. The vectors $\vec{v}_{0}$ and $\vec{v}_{1}$ denote the directional vectors for on- and off-axis ray sets, respectively. The position vector $\vec{w}_{0}$ denotes a fixed point on both plane wave fronts and $n_{2}$ denotes the refractive index of the lens.

Fermat's principle states that the optical path length between two fixed points is an extremum along a light ray. Consider a fixed point on the on-axis wave front defined by $\vec{w}_{0}$ and $\vec{v}_{0}$, and the fixed point $(s(x), f(s(x))$ on the second lens profile: an on-axis ray coming from the wave front and passing through $(s(x), f(s(x))$ must be such that the combined optical path length $d_{1}+d_{2}$ is an extremum. With point $(s(x), f(s(x))$ kept fixed, the only remaining variable to achieve an extremum for $D_{1}=d_{1}+d_{2}$ is the point $(x, f(x))$ on the upper lens profile. Fermat's principle thus implies that

$$
D_{1}=\frac{\partial}{\partial x}\left(d_{1}+d_{2}\right)=0
$$

where the partial derivative indicates that $(s, g(s))$ is kept fixed. Similarly, an on-axis ray between fixed points $(x, f(x))$ and $(0, d)$ must satisfy equation

$$
D_{2}=\frac{\partial}{\partial s}\left(d_{2}+d_{3}\right)=0 .
$$

These two functional differential equations arising from Fermat's principle describe all on-axis ray paths through the lens profiles $f(x)$ and $g(x)$. In analogy, $f(x)$ and $g(x)$ must also satisfy the functional differential equations

$$
\begin{aligned}
& D_{3}=\frac{\partial}{\partial x}\left(\hat{d}_{1}+\hat{d}_{2}\right)=0 \\
& D_{4}=\frac{\partial}{\partial u}\left(\hat{d}_{2}+\hat{d}_{3}\right)=0
\end{aligned}
$$

for off-axis rays, using the same arguments as before. The lens design shown in Fig. 3 is thus fully described by four functional differential equations (4)-(7) for four unknown functions $f(x), g(x), s(x)$ and $u(x)$. The fundamental analysis of a similar system of functional differential equations has been discussed. ${ }^{10-14}$ The results on existence, uniqueness and analyticity were all based on fixed point theorems in functional analysis. These arguments break down when there are off-axis foci. However, the results presented in this work will provide strong evidence that the solutions for off-axis foci are analytic and smooth as well.

Suppose that $(f, g, s, u)$ is an analytic and smooth solution to the functional differential equations (4)-(7), Taylor's theorem implies that the functions must be infinitely differentiable and have a power-series representation. Thus the four functions can be given by power-series

$$
\begin{array}{ll}
f(x)=\sum_{i=0}^{\infty} f_{i}\left(x-x_{0}\right)^{i} & g(x)=\sum_{i=0}^{\infty} g_{i}\left(x-x_{1}\right)^{i} \\
s(x)=\sum_{i=0}^{\infty} s_{i}\left(x-x_{0}\right)^{i} & u(x)=\sum_{i=0}^{\infty} u_{i}\left(x-x_{0}\right)^{i}
\end{array}
$$

centered at convergence points $\left(x_{0}, z_{0}\right)$ and $\left(x_{1}, z_{1}\right)$, respectively. The initial conditions

$$
\begin{array}{ll}
f\left(x_{0}\right)=z_{0} & f^{\prime}\left(x_{0}\right)=m_{0} \\
g\left(x_{1}\right)=z_{1} & g^{\prime}\left(x_{1}\right)=m_{1} \\
s\left(x_{0}\right)=x_{1} & u\left(x_{0}\right)=x_{1}
\end{array}
$$

as introduced in Fig. 3(a) then satisfy the conditional equations $D_{i}=0$ for $i=1 . .4$ and provide general solutions for the initial Taylor coefficients $f_{0}, f_{1}, g_{0}, g_{1}, s_{0}$ and $u_{0}$ depending upon variables $m_{0}$ and $m_{1}$. In ascending order it is now possible to calculate $(\mathrm{n}+1)^{\text {th }}$ order Taylor series coefficients in $f(x)$ and $g(x)$ and $\mathrm{n}^{\text {th }}$ order in $s(x)$ and $u(x)$ by solving equations

$$
\lim _{x \rightarrow x_{0}} \frac{\partial^{n}}{\partial x^{n}} D_{i}=0 \quad(i=1 . .4), \quad\left\{n \in \mathbb{N}_{1}\right\} .
$$

The case for $n=0$ corresponds to the just solved equations for initial Taylor coefficients. There are two further cases needed to be solved: 
1. For $n=1$, the set of equations (11) results in nonlinear algebraic equations for Taylor series coefficients $f_{2}, g_{2}, s_{1}$ and $u_{1}$. These equations have two general solutions, where one solution can be discarded as non-physical. The remaining unique solution can be expressed as functions of the initial, already known Taylor coefficients for $n=0$.

2. For $n>1$, the set of equations (11) results in linear algebraic equations for particular Taylor series coefficients. By sorting and combining terms, the equations (11) can be transformed and expressed as a compact matrix equation

$$
M\left(\begin{array}{c}
f_{n+1} \\
g_{n+1} \\
s_{n} \\
u_{n}
\end{array}\right)=\left(\begin{array}{c}
b_{1}^{(n)} \\
b_{2}^{(n)} \\
b_{3}^{(n)} \\
b_{4}^{(n)}
\end{array}\right)
$$

for arbitrary $n>1$. The matrix elements $M_{i j}$ consist of mathematical expressions which depend on Taylor series coefficients obtained for $n=0,1$. The vector elements of $\vec{b}^{(n)}$ on the right hand side of equation (12) are mathematical expressions only dependent on previous Taylor series coefficients $f_{i}, g_{i}, s_{i-1}$ and $u_{i-1}$ for $i=0 . . n$. Finally, the vector elements of $\vec{b}^{(n)}$ can be calculated for each $n=2,3,4, .$. (in this order) from equation (11). For known matrix $M$ and vectors $\vec{b}^{(2)} . . \vec{b}^{(n)}$, the Taylor series coefficients $f_{n+1}, g_{n+1}, s_{n}$ and $u_{n}$ can then be calculated by solving the linear system of equations (12).

So far, no approximations have been made. The general solutions for $n=0$ and $n=1$ and the introduced algebraic system of linear equations (12) allow to calculate all Taylor series coefficients of $f(x), g(x), s(x)$ and $u(x)$ up to an arbitrary order. However, a Taylor series is a representation of an analytic function as an infinite sum of terms. Indeed, it is only possible to calculate a finite number of initial terms of the Taylor series. Such a function is called a Taylor polynomial and will be the only approximation made. Furthermore, Taylor's remainder theorem provides quantitative estimates on convergence and the approximation error of the function by its Taylor polynomial. The radii of convergence for the expansions $f(x)$ and $g(x)$ are very important, as they indicate the maximum aperture that can be achieved for a given set of initial values. In the examples considered, the radius of convergence is larger than the range of $0<x<x_{\max }$ of the lenses. The algebraic steps of calculation presented in the this section are fully implemented and calculated in Wolfram Mathematica.

\section{EVALUATION OF THE SOLUTION SPACE}

In a first step, the general solutions for Taylor polynomial coefficients for $n=0,1$ are calculated which also determines the matrix $M$ in equation (12). This allows to calculate the general solution vector $\vec{x}$ of the matrix equation $M \vec{x}=\vec{b}$. The vector elements of $\vec{b}^{(n)}$ are calculated within a loop from $n=2$ up to $n=19$, whereas it is possible to calculate even higher orders. All calculated mathematical expressions, sorted in the right order, are then exported as $\mathrm{C}++$ compatible code and embedded in a MATLAB-compatible mex file library. Once compiled, this library returns the Taylor polynomial coefficients for the lens profiles $f(x)$ and $g(x)$ up to $20^{\text {th }}$ order, and the mapping functions $s(x)$ and $u(x)$ up to $19^{\text {th }}$ order as output arguments. The off-axis design angle $\theta$, the refractive index $n_{2}$ and the derivatives at the convergence points $m_{0}$ and $m_{1}$ are passed as input arguments.

For a fixed design angle and refractive index (here: $\theta=14^{\circ}, n_{2}=1.5$ ), the only remaining free parameters to vary are the slopes $m_{0}$ and $m_{1}$. Furthermore, the intended real focus of the lens suggests to choose a negative value for $m_{0}$. The lens' smoothness and symmetry additionally requires $f^{\prime}(0)=g^{\prime}(0)=0$ at the optical axis. This boundary condition introduces an additional correlation between $m_{0}$ and $m_{1}$. Fig. 4(a) shows a contour plot of $\log _{10}\left(\left|f^{\prime}(0)\right|+\left|g^{\prime}(0)\right|\right)$ for slope values $m_{0}<0$ and $-0.1 \leq m_{1} \leq-0.1$ and a step size of $5 \times 10^{-4}$. The logarithmic scale is chosen to better cover the wide range of values.

The contour plot shows that the solutions where $f^{\prime}(0)$ and $g^{\prime}(0)$ are approximately zero lie on a clearly defined curve in $m_{0}$ and $m_{1}$ space. Furthermore, the described surface in parameter space is characterized by a convex curvature which makes it easy to find suitable values using optimization. Fig. 4(b) shows the cross section at a higher resolution (step size $5 \times 10^{-7}$ ) through $m_{0}=-0.32$. The two minima reach down to values below $10^{-8}$. Further quick optimization of $m_{1}$ leads to values well below $10^{-10}$. Fig. 5 shows three different explicit lens solutions for pairs of variates $m_{0}$ and $m_{1}$, marked by crosses in Fig. 4(a). 

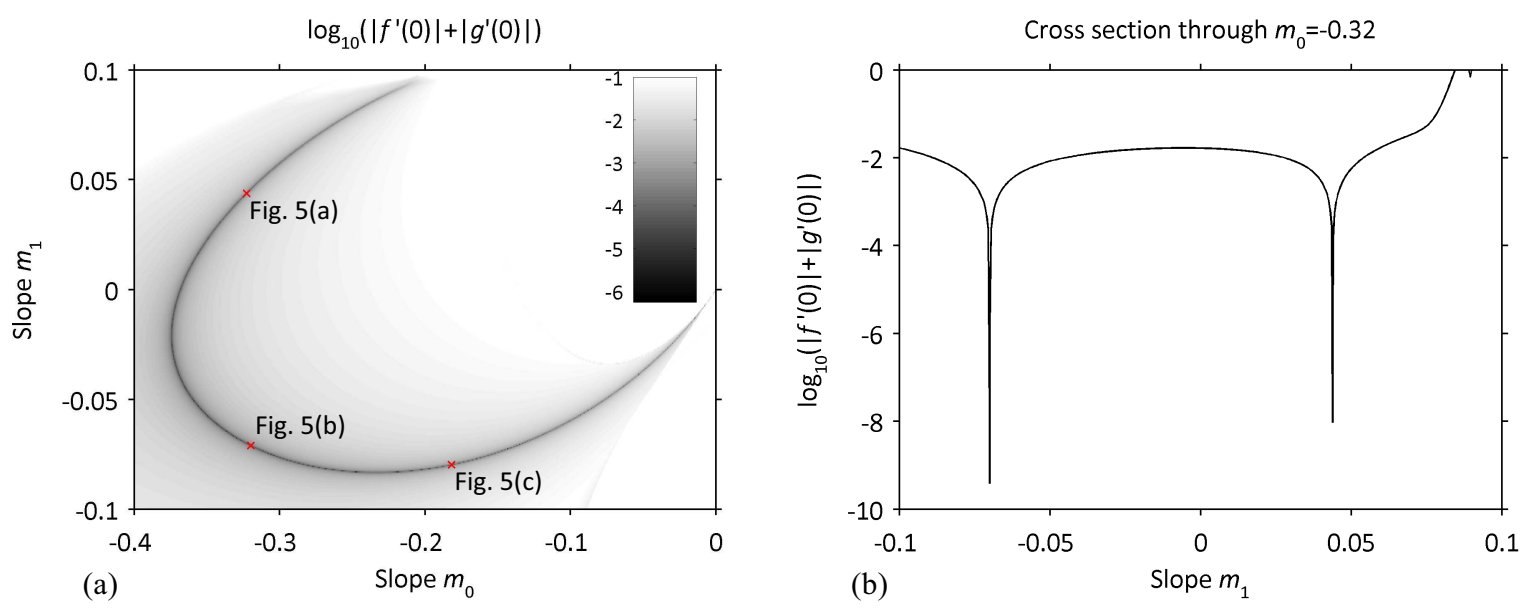

Figure 4. Shown are a contour plot of $\log _{10}\left(\left|f^{\prime}(0)\right|+\left|g^{\prime}(0)\right|\right)$ (a) and the cross section through $m_{0}=-0.32$ (b)

The biconvex lens in Fig. 5(a) is a typical example of a lens design obtained for positive $m_{1}$ values. For negative $m_{1}$ values the solutions provide meniscus lens shapes. Depending on the chosen $m_{0}$ value, the calculated lower lens profile diameter is smaller or bigger than the upper lens profile diameter as shown in Fig. 5(b) and in Fig. 5(c), respectively. These three examples demonstrate the diversity of existing smooth solutions for any pair of variates within the permissible range of values.

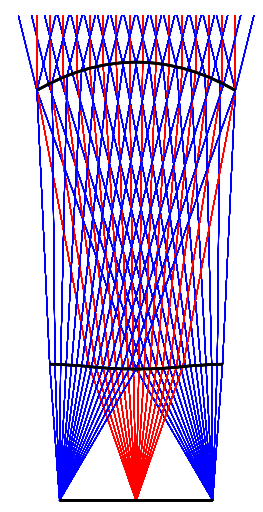

(a)

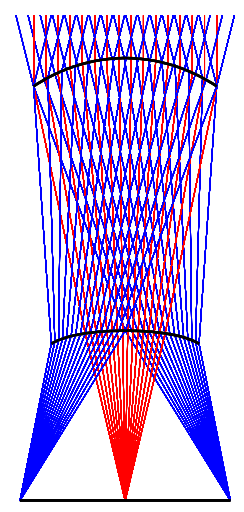

(b)

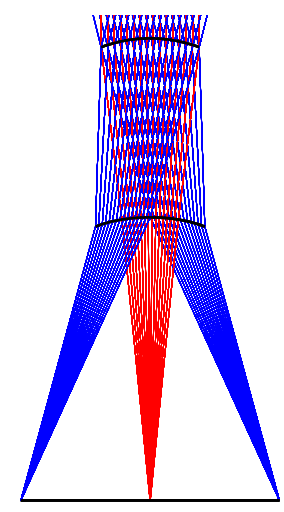

(c)

Figure 5. Three examples of explicit solutions for different pairs of variates $m_{0}$ and $m_{1}$, marked by crosses in Fig. $4(\mathrm{a})$

Even though it is not possible to deduce the correlation $m_{1}\left(m_{0}\right)$ or $m_{0}\left(m_{1}\right)$ as closed form solutions, the numerical calculation of roots of $f^{\prime}(0)$ and $g^{\prime}(0)$ is very accurate and fast. Some more fundamental aspects of this evaluation deserve to be particularly emphasized at the end of this section. The fixed parameters $\theta=14^{\circ}$ and $n_{2}=1.5$ have been selected arbitrarily to analyze the corresponding parameter space and show that it is possible to calculate analytical high order Taylor polynomials for various values $m_{0}$ and $m_{1}$. Consequently, the calculated general solution provides much more. It solves the stated initial segment problem for any given (physically meaningful) initial parameter set $\left(\theta, m_{0}, m_{1}, n_{2}\right)$.

The evaluation for different design angles and refractive indices can be carried out in a similar way. However, the radii of convergence for the expansions $f(x)$ and $g(x)$ should always be taken into account, as they indicate the maximum aperture that can be achieved for a given set of initial values. Taylors remainder theorem provides quantitative estimates on convergence and the approximation error of a function by its Taylor polynomial. 


\section{RAY TRACING ANALYSIS AND CONVERGENCE OF SOLUTIONS}

As discussed in Sec. 2, the introduced analytic design method can be seen as a solution to the initial segment problem of the SMS2D design method. This fact can be used to analyze the overall imaging performance depending on the chosen order for the initial segment curve. The ray tracing simulations in this work are done using the MATLAB-based ray tracer OPS (by courtesy of Prof. Dr. Udo Rohlfing, Hochschule Darmstadt, Germany). As it currently does not provide ray tracing for polynomial functions, $f(x)$ and $g(x)$ are approximated by B-spline curves of $3^{\text {rd }}$ order defined on a grid. To avoid occurring approximation errors in the future, an extension of OPS or the use of a commercial ray tracer will be considered.

To evaluate the overall imaging performance with OPS, the RMS spot radius is analyzed as a function of the incident angle in $x$ - $z$-plane and for monochromatic light (corresponding to $n_{2}=1.5$ ) with the object points at infinity (i.e., parallel rays). Fig. 6(a) and (b) show the RMS spot radius plotted against the incident angle in $0.5^{\circ}$ steps for the biconvex and meniscus lens solutions from Fig. 5(a) and (b). It should be noted that for incident angles beyond $14^{\circ}$, not all rays going through the first lens profile reach the receiver plane anymore.
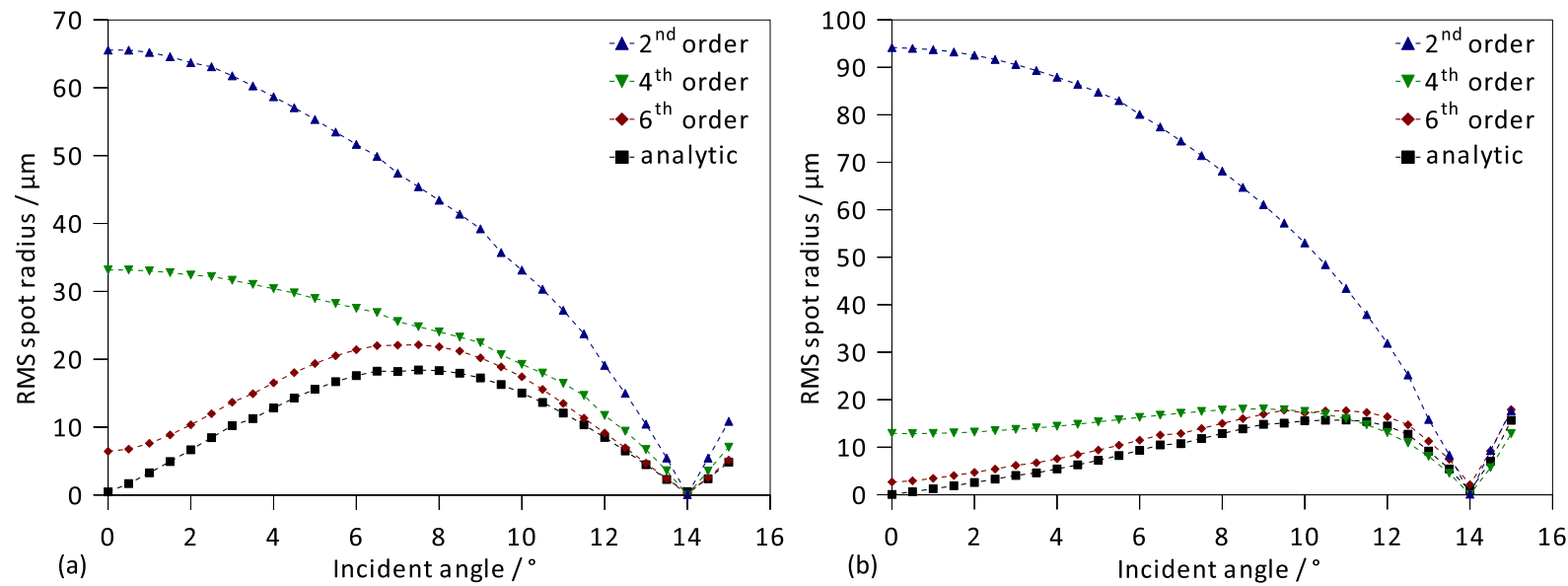

Figure 6. RMS spot radius plotted against the incident angle for the biconvex (a) and the meniscus (b) lens as introduced in Fig. 5(a) and (b) to illustrate how the analytic solution solves asymptotically the stated initial segment problem with increasing order

The procedure is as follows: The known analytic $20^{\text {th }}$ order polynomial solutions for $g(x)$ are approximated by a $2^{\text {nd }}, 4^{\text {th }}$ or $6^{\text {th }}$ order even function, meeting the boundary conditions of the analytic designs. From these approximated lower order initial segments, the SMS2D design method is used to construct the overall lens profiles. The imaging performance of each SMS2D design is then calculated in the same way using OPS, with all compared lenses having an entrance diameter of $3.8 \mathrm{~mm}$. Fig. 6 (a) shows the ray tracing results for the biconvex lens and (b) for the meniscus lens design. Both graphs show very well how the imaging performance converges to the analytic solution with increasing approximation order. As expected, the RMS spot radius at the design angle $14^{\circ}$ is almost zero for all lenses. With increasing approximation order, the RMS spot radius for $0^{\circ}$ tends towards zero. Particularly noteworthy is the fact, that the imaging performance for in-between incident angles is improving as well. The quick convergence of the initial segment approximation should not hide the fact that additional information (such as the positioning and dimensions of the lens profiles) has been already used from the analytic solutions.

These graphs provide a comprehensive quantitative analysis of the convergence of the analytic design method and the link with the SMS2D design method. The here considered single thick lenses are primarily used as a proof of principle. After the successful demonstration of this new optics design method allowing to simultaneously calculate two free-form surfaces, future work will focus on real world applications for which this method can tap its full potential. Therefore, it might be necessary to consider more than two surfaces, different symmetry considerations and generalized wave-fronts for objects at a finite distance. 


\section{CONCLUSIONS}

Within the scope of this work, it has been shown that it is possible to couple three ray sets with two smooth lens profiles forming a lens of minimum thickness. The established convergence point formalism provided the basis for an analytical description of the entire lens by applying Fermat's principle. The derived set of functional differential equations led to an algebraic system of equations which has been solved up to an arbitrary order of all Taylor series coefficients needed to describe the lens profiles. The evaluation of an explicit solution space provided a profound insight into the analytic solution and revealed its covered wide range of possible lens configurations. The ray tracing analysis helped to demonstrate the convergence of this analytic design method as the limiting case of a free-form initial segment to the SMS2D design method. The ray tracing results for analytic lens profiles given as Taylor polynomials of $20^{\text {th }}$ order demonstrated the capabilities and versatility of this new analytic optics design method. Future work will focus on its non-rotational symmetric three-dimensional generalization and target applications where this new design method can help to further increase the overall optical system's performance.

\section{ACKNOWLEDGMENTS}

Work reported in this paper is supported in part by the Research Foundation - Flanders (FWO-Vlaanderen) that provides a $\mathrm{PhD}$ grant (grant number FWOTM510) and provided a grant for a research period at CeDInt, Universidad Politécnica de Madrid (grant number V424711N) for Fabian Duerr, and in part by the IAP BELSPO VI-10, the Industrial Research Funding (IOF), Methusalem, VUB-GOA, and the OZR of the Vrije Universiteit Brussel.

Work is also supported by projects TEC2011-24019 and SEM: TSI-020302-2010-65, funded by the Spanish Ministries MEC and MITYC, respectively.

\section{REFERENCES}

[1] Born, M. and Wolf, E., [Principles of optics], Pergamon Press (1986).

[2] Mouroulis, P. and Macdonald, J., [Geometrical optics and optical design], Oxford University Press, USA (1997).

[3] Chaves, J., [Introduction to nonimaging optics], CRC (2008).

[4] Duerr, F., Benítez, P., Miñano, J. C., Meuret, Y., and Thienpont, H., "Analytic design method for optimal imaging: coupling three ray sets using two free-form lens profiles," Opt. Express 20(5), 5576-5585 (2012).

[5] Grabovičkić, D., Benítez, P., and Miñano, J. C., "Aspheric V-groove reflector design with the SMS method in two dimensions," Opt. Express 18(3), 2515-2521 (2010).

[6] Grabovičkić, D., Benítez, P., and Miñano, J. C., "Free-form V-groove reflector design with the SMS method in three dimensions," Opt. Express 19(104), A747-A756 (2011).

[7] Miñano, J. C., Benítez, P., Lin, W., Infante, J., Muñoz, F., and Santamaría, A., "An application of the SMS method for imaging designs," Opt. Express 17(26), 24036-24044 (2009).

[8] Lin, W., Benítez, P., Miñano, J. C., Infante, J., and Biot, G., "Progress in the SMS design method for imaging optics," Proc. SPIE 8128, 81280F (2011).

[9] Lin, W., Benítez, P., Miñano, J. C., Infante, J., de la Fuente, M. C., and Biot, G., "Solid catadioptric telephoto lens design with SMS method," Proc. SPIE 8167, $81670 \mathrm{O}$ (2011).

[10] Friedman, A. and McLeod, B., "Optimal design of an optical lens," Arch. Rational Mech. Anal. 99(2), 147-164 (1987).

[11] Friedman, A. and McLeod, B., "An optical lens for focusing two pairs of points," Arch. Rational Mech. Anal. 101(1), 57-83 (1988).

[12] Rogers, J. C. W., "Existence, uniqueness, and construction of the solution of a system of ordinary functional differential equations, with application to the design of perfectly focusing symmetric lenses," IMA J. Appl. Math. 41(2), 105 (1988).

[13] Van Brunt, B. and Ockendon, J. R., "A lens focusing light at two different wavelengths," J. Math. Anal. App. 165(1), 156-179 (1992).

[14] Van Brunt, B., "Mathematical possibility of certain systems in geometrical optics," J. Opt. Soc. Am. A 11(11), 2905-2914 (1994). 